\title{
Correlation of common inflammatory cytokines with cognition impairment, anxiety, and depression in acute ischemic stroke patients
}

\author{
Rumei $\mathrm{Li}^{1 \oplus}$, Wenjun Fan ${ }^{2 \odot}$, Dongmei $\mathrm{Li}^{1 \oplus}$, and Xuesong $\mathrm{Liu}^{3 \oplus 凶}$ \\ ${ }^{1}$ Department of Emergency, The Second Affiliated Hospital of Harbin Medical University, Harbin, China \\ ${ }^{2}$ Department of Neurology, The Second Affiliated Hospital of Harbin Medical University, Harbin, China \\ ${ }^{3}$ Nursing Department Office, The Second Affiliated Hospital of Harbin Medical University, Harbin, China
}

\begin{abstract}
Inflammatory cytokines are related to cognitive function and psychiatric disorders in patients with several diseases. However, few relevant studies have been performed on acute ischemic stroke (AIS) patients. Hence, this study aimed to investigate the correlation of common inflammatory cytokines with cognition impairment, anxiety, and depression in AIS patients. Common inflammatory cytokines of 176 AIS patients (including tumor necrosis factor-alpha (TNF- $\alpha$ ), interleukin (IL)-1 $\beta$, IL-6, and IL-17) were measured using Human Enzyme Linked Immunosorbent Assay Kits. Cognition impairment (Mini-Mental State Examination (MMSE)), anxiety (Hospital Anxiety and Depression Scale for anxiety (HADS-A)), and depression (HADS-D) were evaluated. The incidence of cognition impairment, anxiety, and depression was $43.2,39.2$, and $31.2 \%$, respectively. TNF- $\alpha$ and IL- 6 were negatively associated with MMSE score, and high TNF- $\alpha$, IL-1 $\beta$, and IL-6 were correlated with cognition impairment occurrence. In addition, TNF- $\alpha$, IL-1 $\beta$, and IL-17 were positively associated with HADS-A score, while only high TNF- $\alpha$ was associated with anxiety occurrence. Furthermore, TNF- $\alpha$, IL-1 $\beta$, and IL-17 were positively associated with HADS-D score, while high IL-1 $\beta$, IL-6, and IL-17 correlated with depression occurrence. Multivariate logistic regression revealed that TNF- $\alpha$ and National Institutes of Health Stroke Scale (NIHSS) score $\geqslant 5$ were associated with high risk of cognition impairment; TNF- $\alpha$, IL-17, unemployed before surgery, hypertension, and chronic kidney disease (CKD) correlated with high anxiety occurrence. Furthermore, IL-17, divorced/widowed/single status, diabetes, and NIHSS score $\geqslant 5$ were associated with high risk of depression. In conclusion, common inflammatory cytokines including TNF- $\alpha$, IL-1 $\beta$, and IL-17 were related to cognition impairment, anxiety, or depression in AIS patients.
\end{abstract}

Key words: Cognition impairment; Anxiety; Depression; Inflammatory cytokines; Acute ischemic stroke

\section{Introduction}

Stroke is a huge health challenge characterized by the dysfunction and degeneration of brain vascular components, with over 2.5 million new cases in China annually (1). Acute ischemic stroke (AIS) is a major pathological type of stroke and occupies nearly $80 \%$ of new stroke cases $(2,3)$. Although the recent decades have witnessed substantial advances in diagnostic and treatment options, AIS is still the leading cause of disability and one of the leading causes of mortality worldwide (4). Of note, several severe complications often occur to AIS patients, among which, cognitive impairment is a sort of neurological dysfunction manifestation presenting with dysmnesia, disorientation, decline of language competence, and so on, which directly decreases the quality of life of AIS patients $(5,6)$. Other common psychological complications of AIS patients such as anxiety and depression have a negative influence on rehabilitation outcomes, neurotrophic agents' efficacy, and even mortality in AIS patients (7). Therefore, investigating potential indicators related to cognition impairment, anxiety, and depression is a prerequisite for developing effective therapies to improve outcomes of AIS patients.

Inflammation is considered a key step of the progression of ischemic stroke, which has been shown to increase brain injury, retard brain repair, and affect neurological outcomes $(8,9)$. Interestingly, inflammation is also related to cognition impairment, anxiety, and depression in patients with several diseases. For instance, high serum creatinine-reactive protein is associated with depression occurrence in patients with isolated coronary 
artery ectasia (10). Also, increased TNF- $\alpha$ and IL-6 are correlated with high risk of mild cognitive impairment in type 2 diabetes patients (11). However, few relevant studies have been performed in AIS patients. Hence, this study aimed to investigate the correlation of common inflammatory cytokines with cognition impairment, anxiety, and depression in AIS patients.

\section{Material and Methods}

\section{Patients}

This study obtained approval from the Institutional Review Board of The Second Affiliated Hospital of Harbin Medical University. Between January 2019 and July 2020, 176 AIS patients who were admitted to this hospital were consecutively recruited for this study. Eligibility criteria for recruitment were as follows: i) diagnosis of AIS in line with the AIS guideline (12); ii) age more than 18 years; iii) volunteer to participate in the study and provide a blood sample for study use; and iv) able to complete the study assessment. Patients with any of the following conditions were considered ineligible for study enrollment: i) severe cognitive impairment, which was defined as Mini-Mental State Examination (MMSE) score $<10$; ii) presenting with intracranial hemorrhage; iii) known hematological diseases or active infections; iv) administered immunosuppressant within 3 months; v) complicated with inflammatory diseases; vi) had a history of malignancies; and vii) breast feeding or pregnant. All patients signed an informed consent prior to recruitment.

\section{Sample collection and analysis}

Venous blood samples of patients were collected before they were discharged from the hospital after 12-h fasting, and the serum was separated within two hours. After blood collection, the tube was gently inverted and mixed 4-5 times, then put in the upright position at room temperature until the blood was completely coagulated (about $1 \mathrm{~h}$ ). Following that, centrifugation was conducted at $1000 \mathrm{~g}$ for $10 \mathrm{~min}$ at room temperature, then serum was obtained. The collected serum was transferred to cryopreserved tubes and placed in $\mathrm{a}-70^{\circ} \mathrm{C}$ refrigerator. Levels of inflammatory cytokines in serum, including TNF- $\alpha$, interleukin (IL)-1 $\beta$, IL-6, and IL-17, were measured using Human Enzyme Linked Immunosorbent Assay (ELISA) kits (Invitrogen, USA). The ELISA was carried out following the manufacturer's protocol.

\section{Data collection and evaluation}

Clinical data collection was completed during hospitalization of patients, which mainly included sociodemographic information, smoking status, complications, as well as disease-related features. Assessment of cognition impairment, anxiety, and depression was conducted for all patients on the day of discharge. The cognition impairment status of patients was evaluated using MMSE, and a
MMSE score $\leqslant 26$ was considered as cognition impairment (13). The anxiety status and depression status of patients were assessed using the Hospital Anxiety and Depression Scale for anxiety (HADS-A) and the Hospital Anxiety and Depression Scale for depression (HADS-D), respectively. An HADS-A score $>7$ was indicative of anxiety, and similarly, a HADS-D score $>7$ was indicative of depression. Furthermore, the anxiety degree was classified as 8-10, mild anxiety; 11-14, moderate anxiety; and 15-21, severe anxiety (14). The depression degree was classified as 8-10, mild depression; 11-14, moderate depression; and 15-21, severe depression (14).

\section{Statistical analysis}

SPSS 24.0 (IBM, USA) and GraphPad Prism 7.02 software (GraphPad Software Inc., USA) were applied for data analysis and diagram making. Descriptive analysis was performed for characteristics of patients, MMSE score, HADS-A score, and HADS-D score. Correlation analysis was determined by Spearman's rank correlation test. Comparison of differences was determined by chisquared test or Wilcoxon rank sum test, as appropriate. All potential factors were included in the multivariate logistic regression analysis of cognition impairment, anxiety, and depression, and the independent factors were screened out by forward stepwise (conditional) method. A significant difference was indicated by a $\mathrm{P}$ value $<0.05$.

\section{Results}

\section{AIS patients' characteristics}

The detailed information about other characteristics is shown in Table 1. The mean age was $67.6 \pm 8.4$ years, and there were $63.1 \%$ males and $36.9 \%$ females. With respect to marriage status, $47.2 \%$ patients were married and $52.8 \%$ patients were divorced/widowed/single. As for employment status before AIS, $11.4 \%$ patients were employed and $88.6 \%$ patients were unemployed. In addition, $27.3 \%$ patients were current smokers, $84.7 \%$ patients had hypertension, $50.0 \%$ patients had hyperlipidemia, $36.4 \%$ patients had diabetes mellitus, and $13.6 \%$ patients had CKD. Furthermore, 40.3, 34.1, and $25.6 \%$ of patients had left, right, and bilateral brainstem unknown lesion location, respectively. The mean NIHSS score was $7.0 \pm 3.0$.

\section{Cognition impairment, anxiety, and depression assessment}

In regard to cognition impairment, the mean value, median value, and the range of MMSE were $26.5 \pm 1.9$, 27.0 (IQR: 25.0-28.0), and 22.0-30.0, respectively (Figure 1A). There were $43.2 \%$ patients with cognition impairment, and $56.8 \%$ patients with no cognition impairment (Figure 1B). Regarding anxiety, the mean value, median value, and the range of HADS-A were $7.1 \pm 3.4$, 6.0 (IQR: 4.0-9.0), and 1.0-17.0, respectively (Figure 1C). 
Table 1. Characteristics of acute ischemic stroke (AIS) patients.

\begin{tabular}{|c|c|}
\hline Items & AIS patients $(n=176)$ \\
\hline Age (years), mean $\pm \mathrm{SD}$ & $67.6 \pm 8.4$ \\
\hline \multicolumn{2}{|l|}{ Gender, n (\%) } \\
\hline Male & $111(63.1)$ \\
\hline Female & $65(36.9)$ \\
\hline Education duration (years), median (IQR) & $7.0(5.3-9.0)$ \\
\hline \multicolumn{2}{|l|}{ Marriage status, n (\%) } \\
\hline Married & $83(47.2)$ \\
\hline Divorced/widowed/single & $93(52.8)$ \\
\hline \multicolumn{2}{|l|}{ Employment status before AIS, n (\%) } \\
\hline Employed & $20(11.4)$ \\
\hline Unemployed & $156(88.6)$ \\
\hline \multicolumn{2}{|l|}{ Current smoking, $\mathrm{n}(\%)$} \\
\hline No & $128(72.7)$ \\
\hline Yes & $48(27.3)$ \\
\hline \multicolumn{2}{|l|}{ Hypertension, n (\%) } \\
\hline No & $27(15.3)$ \\
\hline Yes & $149(84.7)$ \\
\hline \multicolumn{2}{|l|}{ Hyperlipidemia, n (\%) } \\
\hline No & $88(50.0)$ \\
\hline Yes & $88(50.0)$ \\
\hline \multicolumn{2}{|l|}{ Diabetes mellitus, $\mathrm{n}(\%)$} \\
\hline No & $112(63.6)$ \\
\hline Yes & $64(36.4)$ \\
\hline \multicolumn{2}{|l|}{ CKD, n (\%) } \\
\hline No & $152(86.4)$ \\
\hline Yes & $24(13.6)$ \\
\hline \multicolumn{2}{|l|}{ Lesion location, n (\%) } \\
\hline Left & $71(40.3)$ \\
\hline Right & $60(34.1)$ \\
\hline Bilateral/brainstem/unknown & $45(25.6)$ \\
\hline NIHSS score ${ }^{*}$, mean \pm SD & $7.0 \pm 3.0$ \\
\hline
\end{tabular}

SD: standard deviation; IQR: interquartile range; CKD: chronic kidney disease; NIHSS: National Institutes of Health Stroke Scale. *NIHSS score was evaluated within $24 \mathrm{~h}$ after admission.

There were $60.8,22.7,13.1$, and $3.4 \%$ of patients with a grade of no anxiety, mild anxiety, moderate anxiety, and severe anxiety (Figure 1D). In addition, the mean value, median value, and the range of HADS-D were $6.7 \pm 3.0$, 6.0 (IQR: 5.0-8.0), and 1.0-16.0, respectively (Figure 1E); There were $68.8,19.3,9.6$, and $2.3 \%$ of patients with no depression, mild depression, moderate depression, and severe depression, respectively (Figure 1F).

Characteristics associated with cognition impairment, anxiety, and depression

Cognition impairment occurrence was correlated with older age $(P=0.035)$, hyperlipidemia $(P=0.033)$, and NIHSS score $\geqslant 5(P<0.001)$. Furthermore, anxiety occurrence was associated with divorced/widowed/single status $(P=$ 0.043), unemployed status $(P=0.019)$, hypertension $(P=$ $0.005)$, diabetes mellitus $(P<0.001)$, and CKD $(P=0.012)$. In addition, depression occurrence was related to divorced/ widowed/single status $(P=0.010)$, diabetes mellitus $(P=$ $0.001)$, CKD $(P=0.009)$, and high NIHSS score $(P=0.008)$ (Table 2).

\section{Correlation of inflammatory cytokines with cognition impairment}

TNF- $\alpha(P<0.001)$ and IL-6 $(P=0.002)$, but not IL-1 $\beta$ $(P=0.069)$ or IL-17 $(P=0.374)$, were negatively associated with MMSE score. In addition, high TNF- $\alpha(P<0.001$, adjusted $P<0.001)$, IL-1 $\beta(P=0.033$, adjusted $P=0.132)$, and IL-6 $(P=0.012$, adjusted $P=0.048)$, but not $\mathrm{IL}-17$ $(\mathrm{P}=0.554, \mathrm{P}=1.000$ after adjustment) were correlated with cognition impairment occurrence (Figure 2A-H).

\section{Correlation of inflammatory cytokines with anxiety}

TNF- $\alpha \quad(P=0.001), I L-1 \beta \quad(P=0.046)$, and IL-17 $(P=$ $0.013)$, but not IL-6 $(P=0.242)$, were positively associated with HADS-A score. High TNF- $\alpha(P=0.002$, adjusted $P=0.008)$, but not high IL-1 $\beta \quad(P=0.864$, adjusted $P=1.000)$, IL-6 $(P=0.160$, adjusted $P=0.640)$, and $I L-17$ $(P=0.266$, adjusted $P=1.000)$, was correlated with anxiety occurrence. Furthermore, high TNF- $\alpha(\mathrm{P}<0.001$, adjusted $P<0.001)$ and increased IL-17 $(P=0.014$, adjusted $P=$ $0.056)$, but not $\mathrm{IL}-1 \beta(\mathrm{P}=0.648$, adjusted $\mathrm{P}=1.000)$ or IL-6 ( $P=0.131$, adjusted $P=0.524)$, were correlated with increased anxiety severity grade (Figure $3 A-L$ ).

\section{Correlation of inflammatory cytokines with depression}

TNF- $\alpha \quad(P=0.005), \quad I L-1 \beta \quad(P<0.001), \quad$ and $\quad \mathrm{IL}-17$ $(P=0.024)$, but not IL-6 $(P=0.165)$, were positively associated with HADS-D score. High IL-1 $\beta(P=0.011$, adjusted $\mathrm{P}=0.044)$, IL-6 $(\mathrm{P}=0.010$, adjusted $\mathrm{P}=0.040)$, and $\mathrm{IL}-17$ ( $P=0.011, P=0.044$ after adjustment), but not TNF- $\alpha$ ( $P=0.104, P=0.416$ after adjustment), were associated with depression occurrence. Furthermore, high TNF- $\alpha$ $(P=0.044, P=0.176$ after adjustment), high $\mathrm{IL}-1 \beta(P=0.012$, $P=0.048$ after adjustment), increased IL-6 $(P=0.010$, $\mathrm{P}=0.040$ after adjustment), and increased IL-17 ( $\mathrm{P}=$ $0.010, P=0.040$ after adjustment) were correlated with increased depression severity grade (Figure 4A-L).

\section{Independent risk factors related to cognition impairment, anxiety, and depression}

Multivariate logistic regression showed that TNF- $\alpha$ $(\mathrm{OR}=2.882, \mathrm{P}=0.004)$ and NIHSS score $\geqslant 5(\mathrm{OR}=1.009$, $\mathrm{P}=0.004)$ were associated with high risk of cognition impairment. TNF- $\alpha$ (OR=1.007, $P=0.025), \quad I L-17$ (OR= $1.008, P=0.007)$, unemployed before surgery $(\mathrm{OR}=4.099$, $\mathrm{P}=0.046$ ), hypertension (OR=5.283, $\mathrm{P}=0.007$ ), and CKD $(\mathrm{OR}=3.224, \mathrm{P}=0.026)$ were associated with high risk of anxiety. Furthermore, IL-17 (OR=1.007, $P=0.009)$, divorced/widowed/single status ( $O R=2.668, P=0.008)$, diabetes $(\mathrm{OR}=3.337, \mathrm{P}=0.001)$, and NIHSS score $\geqslant 5$ $(\mathrm{OR}=2.370, \mathrm{P}=0.036)$ were associated with high risk of depression (Table 3 ). 
A

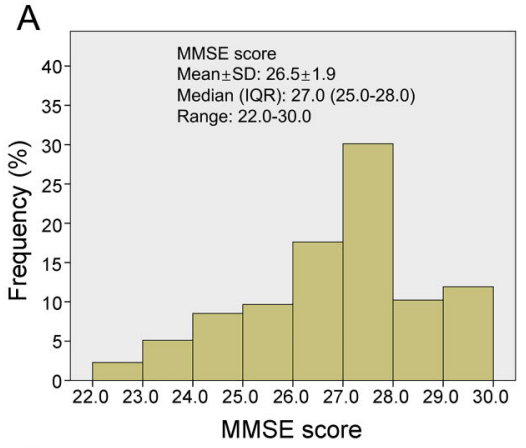

$\mathrm{B}$

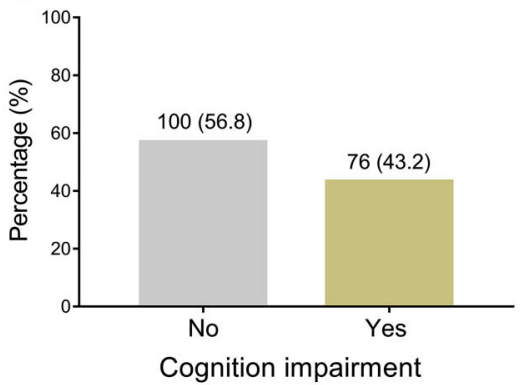

C

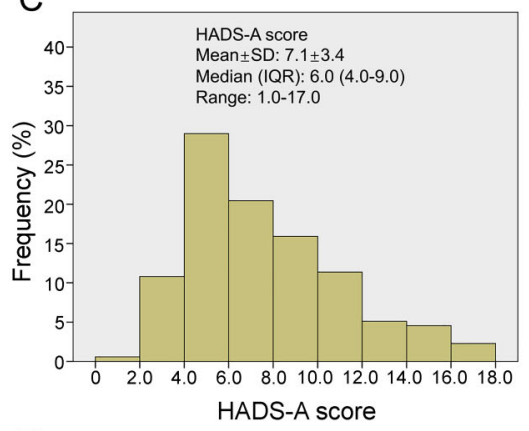

D

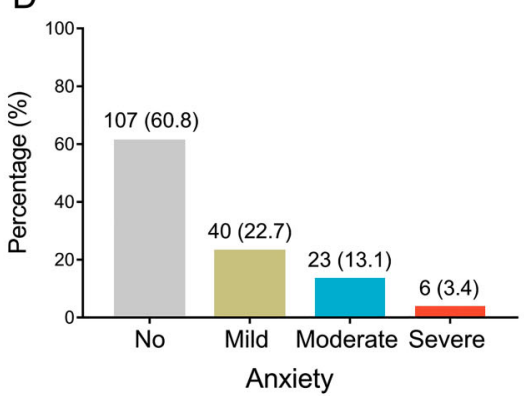

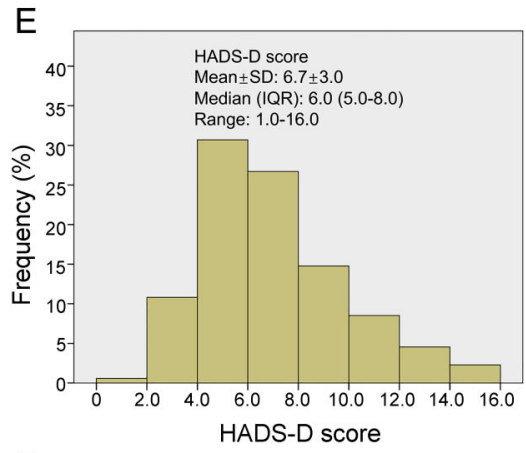

$\mathrm{F}$

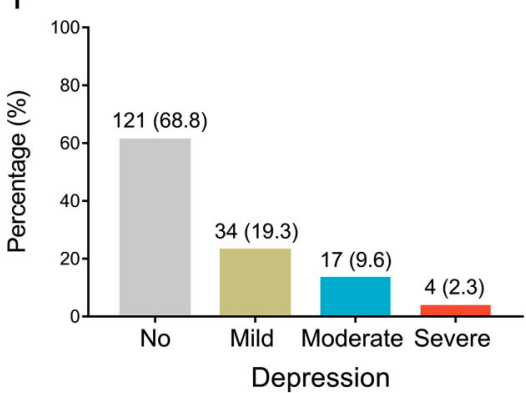

Figure 1. Overall cognition impairment, anxiety, and depression in acute ischemic stroke patients. Distribution of MMSE score (A), HADS-A score (C), and HADS-D score (E). Number (percentage) of cognition impairment (B), anxiety (D), and depression (F) grade. MMSE: Mini-Mental State Examination; HADS-A: Hospital Anxiety and Depression Scale for anxiety; HADS-D: Hospital Anxiety and Depression Scale for depression; SD: standard deviation; IQR: interquartile range.

\section{Discussion}

In the current study, the incidence of cognition impairment, anxiety, and depression was 43.2, 39.2, and $31.2 \%$ in AIS patients. After acute ischemic insult, inflammatory cytokines in the ischemic brain will be upregulated from resident brain cells and infiltrating immune cells, which play complex roles in the pathophysiology of cerebral ischemia (9). Previous evidence supports a relationship of cognition impairment, anxiety, and depression with inflammation processes in several diseases (such as coronary heart patients and cancer) $(10,15,16)$. However, few studies have been performed on the potential cognition impairment/anxiety/depression-associated inflammatory cytokines in AIS patients. Hence, deeply understanding the clinical implication of inflammatory cytokines on cognition impairment/anxiety/depression is a precondition to improve the prognosis of AIS patients. In the present study, we discovered that high TNF- $\alpha$ and IL-6 were correlated with cognition impairment occurrence; high TNF- $\alpha$, IL-1 $\beta$, and IL-17 were correlated with anxiety or depression occurrence in AIS patients. The possible reasons were that: 1) TNF- $\alpha$ and IL-6, as pro-inflammatory cytokines, could cross the blood-brain barrier by a transport system, thereby promoting communication between the central nervous system and the periphery $(17,18)$. Hence, high concentrations of TNF- $\alpha$ and IL-6 might be important factors for promoting the development of cognition impairment, thereby increased cognition impairment occurrence; and 2) a high level of common pro-inflammatory cytokines (including TNF- $\alpha, \mathrm{IL}-1 \beta$, and IL-17) might affect indoleamine 2,3-deoxygenation enzyme-1 (IDO1), subsequently influence 5-hydroxytryptamine (5-HT) (an important neurotransmitter related to depression), and eventually increase anxiety and depression occurrence in AIS patients (19-22).

In addition, TNF- $\alpha$ and NIHSS score $\geqslant 5$ were related to a high risk of cognition impairment. Also, IL-17, unemployed before surgery, hypertension, CKD, divorced/ widowed/single status, diabetes, and NIHSS score $\geqslant 5$ were correlated with a high risk of anxiety or depression in AIS patients. The probable explanations were as follows: 1) Regarding cognition impairment, TNF- $\alpha$ served as a common inflammation cytokine, and the possible reason for its correlation with cognition impairment was as mentioned above; high NIHSS score meant worse neurological status in AIS patients, and cognition impairment was also a kind of manifestation of neurological dysfunction, hence, an NIHSS score $\geqslant 5$ was an independent risk factor for cognition impairment. 2) As for anxiety and depression, the impact of IL-17 on anxiety and depression was also as mentioned above. Furthermore, unemployed before surgery and divorced/widowed/single status meant unfavorable social status, which might make patients feel less capable 
Table 2. Cognition impairment, anxiety, and depression rate among acute ischemic stroke (AIS) patients with different clinical features.

\begin{tabular}{|c|c|c|c|}
\hline Clinical features & Cognition impairment & Anxiety & Depression \\
\hline \multicolumn{4}{|l|}{ Age, n (\%) } \\
\hline$<70$ years & $35(36.1)$ & $34(35.1)$ & $28(28.9)$ \\
\hline$\geqslant 70$ years & $41(51.9)$ & $35(44.3)$ & $27(34.2)$ \\
\hline$P$ value & 0.035 & 0.211 & 0.450 \\
\hline \multicolumn{4}{|l|}{ Gender, n (\%) } \\
\hline Male & $53(47.7)$ & $42(37.8)$ & $36(32.4)$ \\
\hline Female & $23(35.4)$ & $27(41.5)$ & $19(29.2)$ \\
\hline$P$ value & 0.110 & 0.627 & 0.658 \\
\hline \multicolumn{4}{|l|}{ Education duration, $\mathrm{n}(\%)$} \\
\hline$<7$ years & $35(43.2)$ & $28(34.6)$ & $21(25.9)$ \\
\hline$\geqslant 7$ years & $41(43.2)$ & $41(43.2)$ & $34(35.8)$ \\
\hline$P$ value & 0.994 & 0.245 & 0.159 \\
\hline \multicolumn{4}{|l|}{ Marriage status, n (\%) } \\
\hline Married & $34(41.0)$ & $26(31.3)$ & $18(21.7)$ \\
\hline Divorced/widowed/single & $42(45.2)$ & $43(46.2)$ & $37(39.8)$ \\
\hline$P$ value & 0.159 & 0.043 & 0.010 \\
\hline \multicolumn{4}{|c|}{ Employment status before AIS, $\mathrm{n}(\%)$} \\
\hline Employed & $7(35.0)$ & $3(15.0)$ & $5(25.0)$ \\
\hline Unemployed & $69(44.2)$ & $66(42.3)$ & $50(32.1)$ \\
\hline$P$ value & 0.433 & 0.019 & 0.522 \\
\hline \multicolumn{4}{|l|}{ Current smoking, $\mathrm{n}(\%)$} \\
\hline No & $60(46.9)$ & $54(42.2)$ & $42(32.8)$ \\
\hline Yes & $16(33.3)$ & $15(31.3)$ & $13(27.1)$ \\
\hline$P$ value & 0.106 & 0.186 & 0.465 \\
\hline \multicolumn{4}{|l|}{ Hypertension, n (\%) } \\
\hline No & $8(29.6)$ & $4(14.8)$ & $5(18.5)$ \\
\hline Yes & $68(45.6)$ & $65(43.6)$ & $50(33.6)$ \\
\hline$P$ value & 0.122 & 0.005 & 0.121 \\
\hline \multicolumn{4}{|l|}{ Hyperlipidemia, n (\%) } \\
\hline No & $31(35.2)$ & $35(39.8)$ & $26(29.5)$ \\
\hline Yes & $45(51.1)$ & $34(38.6)$ & $29(33.0)$ \\
\hline$P$ value & 0.033 & 0.877 & 0.626 \\
\hline \multicolumn{4}{|l|}{ Diabetes mellitus, n (\%) } \\
\hline No & $45(40.2)$ & $33(29.5)$ & $25(22.3)$ \\
\hline Yes & $31(48.4)$ & $36(56.3)$ & $30(46.9)$ \\
\hline$P$ value & 0.287 & $<0.001$ & 0.001 \\
\hline \multicolumn{4}{|l|}{ CKD, n (\%) } \\
\hline No & $68(44.7)$ & $54(35.5)$ & $42(27.6)$ \\
\hline Yes & $8(33.3)$ & $15(62.5)$ & $13(54.2)$ \\
\hline$P$ value & 0.295 & 0.012 & 0.009 \\
\hline \multicolumn{4}{|l|}{ Lesion location, n (\%) } \\
\hline Left & $30(42.3)$ & $31(43.7)$ & $23(32.4)$ \\
\hline Right & $28(46.7)$ & $21(35.0)$ & $19(31.7)$ \\
\hline Bilateral/brainstem/unknown & $18(40.0)$ & $17(37.8)$ & $13(28.9)$ \\
\hline$P$ value & 0.776 & 0.584 & 0.921 \\
\hline \multicolumn{4}{|l|}{ NIHSS score*, n (\%) } \\
\hline$<5$ & $14(23.3)$ & $18(30.0)$ & $11(18.3)$ \\
\hline$\geqslant 5$ & $62(53.4)$ & $51(44.0)$ & $44(37.9)$ \\
\hline$P$ value & $<0.001$ & 0.072 & 0.008 \\
\hline
\end{tabular}

CKD: chronic kidney disease; NIHSS: National Institutes of Health Stroke Scale. *NIHSS score was evaluated within $24 \mathrm{~h}$ after admission. Bold type indicates statistical significance (chi-squared test or Wilcoxon rank sum test). 
A
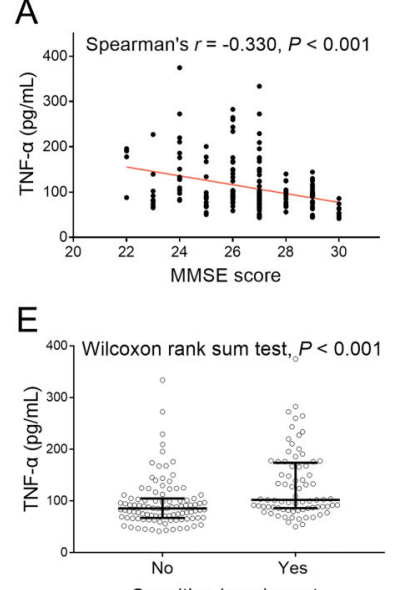

$\mathrm{B}$

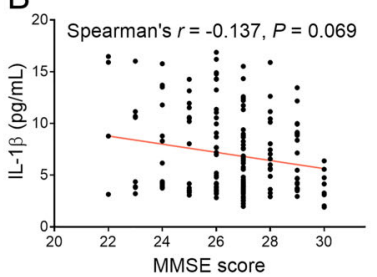

$F$

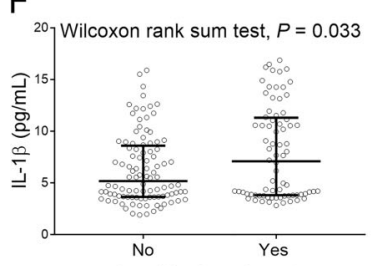

C

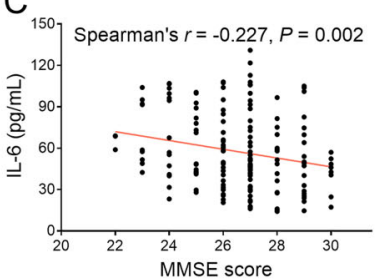

G

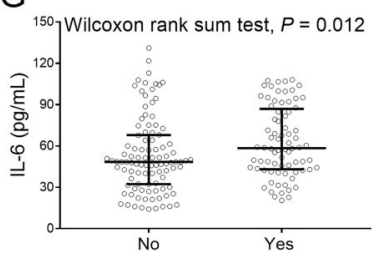

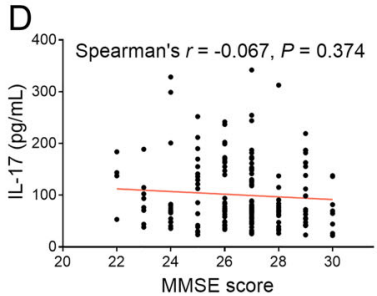

$\mathrm{H}$

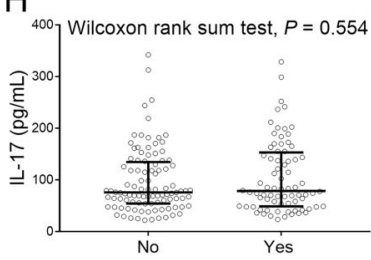

Figure 2. Common inflammatory cytokines were positively associated with cognition impairment in acute ischemic stroke patients. Correlation of TNF- $\alpha$, IL-1 $\beta$, IL-6, and IL-17 with MMSE score (A-D) and with cognition impairment occurrence (E-H). Lines indicate median and IQR. TNF- $\alpha$ : tumor necrosis factor alpha; IL: interleukin; MMSE: Mini-Mental State Examination.

A

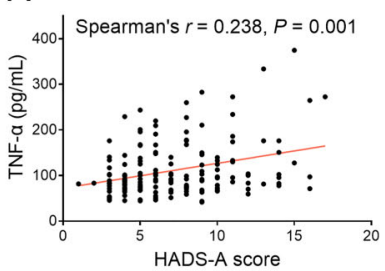

E

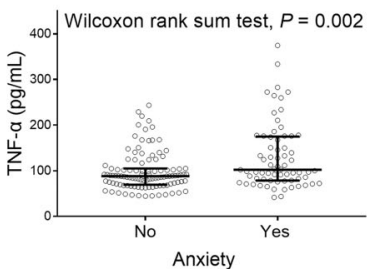

I

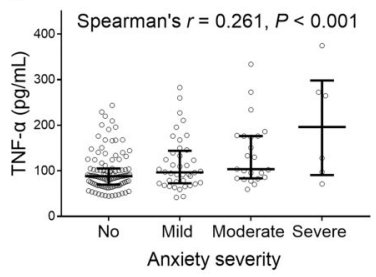

B

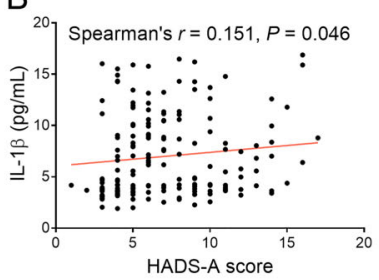

F

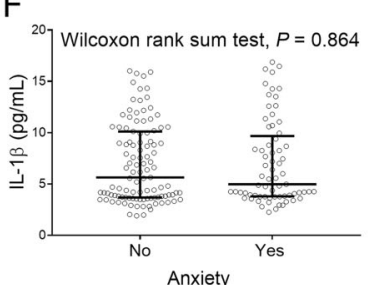

J

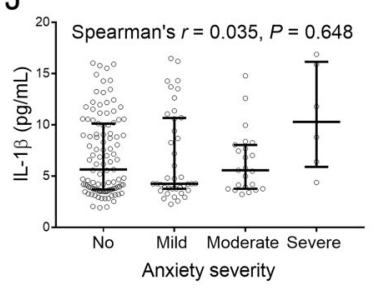

C

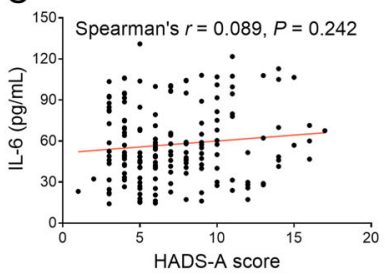

G

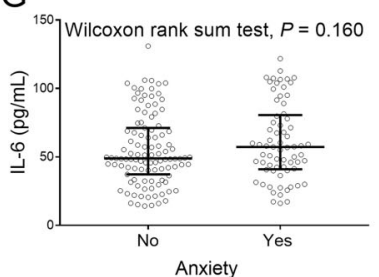

K

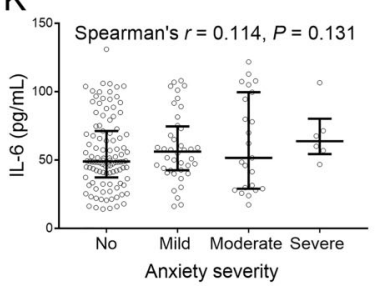

D

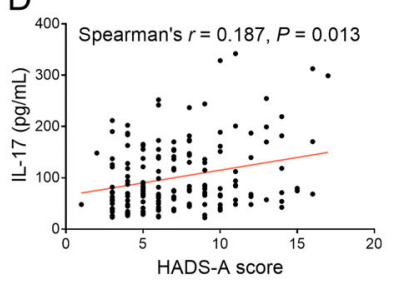

$\mathrm{H}$

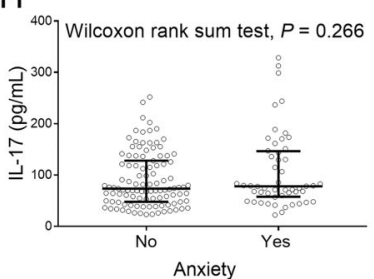

L

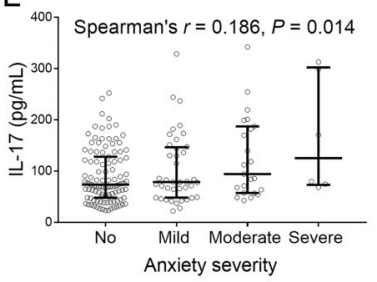

Figure 3. Common inflammatory cytokines were positively associated with anxiety in acute ischemic stroke patients. Correlation of TNF- $\alpha$, IL-1 $\beta$, IL-6, and IL-17 with HADS-A (A-D), with anxiety occurrence (E-H), and with anxiety severity (I-L). Lines indicate median and IQR. TNF- $\alpha$ : tumor necrosis factor alpha; IL: interleukin; HADS-A: Hospital Anxiety and Depression Scale for anxiety.

and alone, increasing stress after AIS, thereby causing a high risk of anxiety and depression. Meanwhile, patients with severe complications (including hypertension, CKD, and diabetes) are under long-term metabolic dysfunction, which might affect their immune system and increase their negative stress, thereby leading to a high risk of anxiety and depression in AIS patients. In addition, an NIHSS score $\geqslant 5$ meant worse neurological status in AIS patients, which also produces huge stress, and eventually, increased anxiety and depression occurrence in AIS patients. 
A

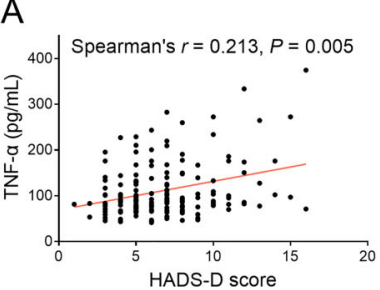

E

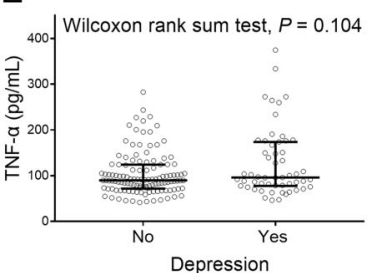

I

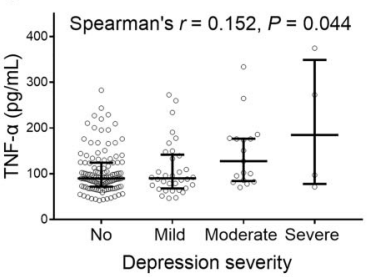

B

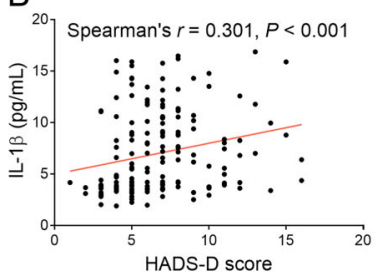

F

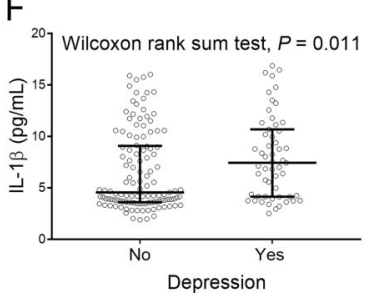

$J$

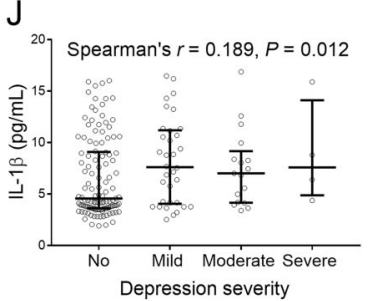

C

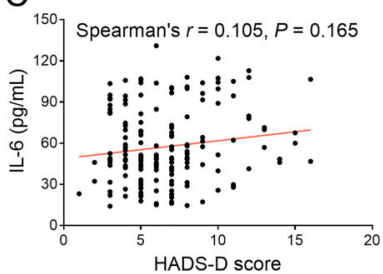

G
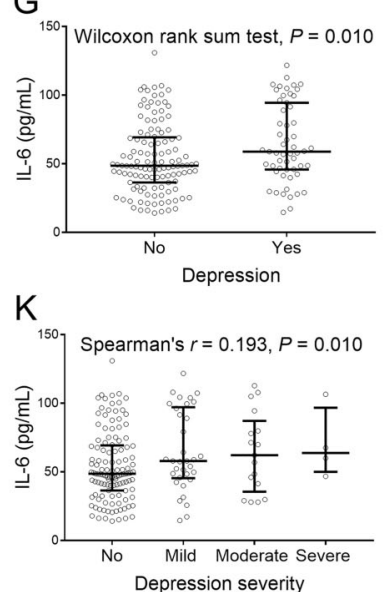

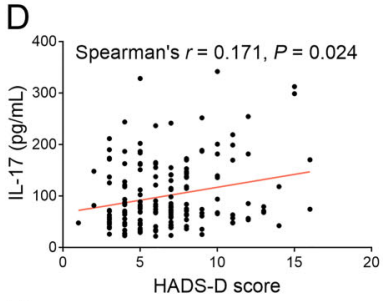

$\mathrm{H}$

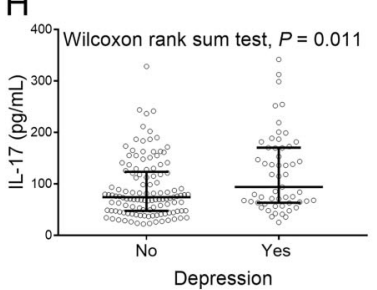

L

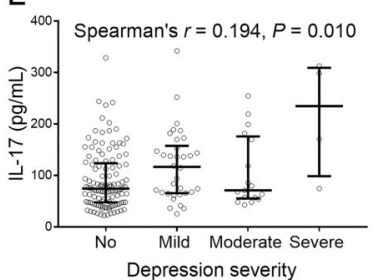

Figure 4. Common inflammatory cytokines were positively associated with depression in acute ischemic stroke patients. Correlation of TNF- $\alpha$, IL-1 $\beta$, IL-6, and IL-17 with HADS-A (A-D), with depression occurrence (E-H), and with depression severity (I-L). Lines indicate median and IQR. TNF- $\alpha$ : tumor necrosis factor alpha; IL: interleukin; HADS-D: Hospital Anxiety and Depression Scale for depression.

Table 3. Independent factors associated with cognition impairment, anxiety, and depression of acute ischemic stroke (AIS) patients.

\begin{tabular}{|c|c|c|c|c|c|c|}
\hline \multirow[t]{3}{*}{ Parameters } & \multicolumn{6}{|c|}{ Multivariate logistic regression\# } \\
\hline & \multirow[t]{2}{*}{ SE } & \multirow[t]{2}{*}{ Wald } & \multirow[t]{2}{*}{$P$ value } & \multirow[t]{2}{*}{ OR } & \multicolumn{2}{|c|}{$95 \% \mathrm{Cl}$} \\
\hline & & & & & Lower & Upper \\
\hline \multicolumn{7}{|l|}{ Cognition impairment } \\
\hline TNF- $\alpha$ & 0.371 & 8.161 & 0.004 & 2.882 & 1.394 & 5.958 \\
\hline NIHSS ${ }^{*}$ score $\geqslant 5$ ( $<5$ as ref.) & 0.003 & 8.080 & 0.004 & 1.009 & 1.003 & 1.016 \\
\hline \multicolumn{7}{|l|}{ Anxiety } \\
\hline TNF- $\alpha$ & 0.003 & 5.042 & 0.025 & 1.007 & 1.001 & 1.014 \\
\hline IL-17 & 0.003 & 7.168 & 0.007 & 1.008 & 1.002 & 1.014 \\
\hline Unemployed before surgery (Employed as ref.) & 0.706 & 3.997 & 0.046 & 4.099 & 1.028 & 16.345 \\
\hline Hypertension (No hypertension as ref.) & 0.621 & 7.173 & 0.007 & 5.283 & 1.563 & 17.861 \\
\hline CKD (no CKD as ref.) & 0.527 & 4.927 & 0.026 & 3.224 & 1.147 & 9.065 \\
\hline \multicolumn{7}{|l|}{ Depression } \\
\hline IL-17 & 0.003 & 6.867 & 0.009 & 1.007 & 1.002 & 1.013 \\
\hline Divorced/widowed/single (married as ref.) & 0.372 & 6.941 & 0.008 & 2.668 & 1.286 & 5.537 \\
\hline Diabetes (no diabetes as ref.) & 0.366 & 10.869 & 0.001 & 3.337 & 1.630 & 6.832 \\
\hline NIHSS score ${ }^{*} \geqslant 5(<5$ as ref. $)$ & 0.412 & 4.390 & 0.036 & 2.370 & 1.057 & 5.314 \\
\hline
\end{tabular}

SE: standard error; OR: odds ratio; Cl: confidence interval; TNF- $\alpha$ : tumor necrosis factor alpha; NIHSS: National Institutes of Health Stroke Scale; IL-17: interleukin 17; CKD: chronic kidney disease. *NIHSS score was evaluated within $24 \mathrm{~h}$ after admission. ${ }^{*}$ All potential factors were included in the multivariate logistic regression analysis, and the independent factors were screened out by forward stepwise (conditional) method. 
Several limitations existed in this study. Although the potential cognition impairment/anxiety/depression-associated inflammatory cytokines in AIS patients have been explored, the detailed mechanisms of these inflammatory cytokines underlying cognition impairment/anxiety/

\section{References}

1. Sun H, Zou X, Liu L. Epidemiological factors of stroke: a survey of the current status in China. J Stroke 2013; 15: 109-114, doi: 10.5853/jos.2013.15.2.109.

2. Khandelwal $P$, Yavagal DR, Sacco RL. Acute ischemic stroke intervention. J Am Coll Cardiol 2016; 67: 2631-2644, doi: 10.1016/j.jacc.2016.03.555.

3. Carota A, Dieguez S, Bogousslavsky J. Psychopathology of stroke [in French]. Psychol Neuropsychiatr Vieil 2005; 3: 235-249, doi: 10.1590/1414-431X20198533.

4. Phipps MS, Cronin CA. Management of acute ischemic stroke. BMJ 2020; 368: I6983, doi: 10.1136/bmj.16983.

5. Pendlebury ST, Rothwell PM. Prevalence, incidence, and factors associated with pre-stroke and post-stroke dementia: a systematic review and meta-analysis. Lancet Neurol 2009; 8: 1006-1018, doi: 10.1016/S1474-4422(09)70236-4.

6. Zhang L, Zhang T, Sun Y. A newly designed intensive caregiver education program reduces cognitive impairment, anxiety, and depression in patients with acute ischemic stroke. Braz J Med Biol Res 2019; 52: e8533, doi: 10.1590/ 1414-431x20198533.

7. Li W, Xiao WM, Chen YK, Qu JF, Liu YL, Fang XW, et al. Anxiety in patients with acute ischemic stroke: risk factors and effects on functional status. Front Psychiatry 2019; 10: 257, doi: 10.3389/fpsyt.2019.00257.

8. Yilmaz G, Granger DN. Cell adhesion molecules and ischemic stroke. Neurol Res 2008; 30: 783-793, doi: 10.1179/ $174313208 \times 341085$

9. Jin R, Liu L, Zhang S, Nanda A, Li G. Role of inflammation and its mediators in acute ischemic stroke. $J$ Cardiovasc Trans/ Res 2013; 6: 834-851, doi: 10.1007/s12265-0139508-6.

10. Gurbuz AS, Alsancak Y, Sakli B, Duzenli MA. Association between depression and anxiety scores and inflammation in patients with isolated coronary artery ectasia. Turk Kardiyol Dern Ars 2019; 47: 365-372, doi: 10.5543/tkda.2019.45403.

11. Huang HH, Chang JC, Liu HC, Yang ZY, Yang YJ, Chen LK, et al. Handgrip strength, tumor necrosis factor-alpha, interlukin-6, and visfatin levels in oldest elderly patients with cognitive impairment. Exp Gerontol 2020; 142: 111138, doi: 10.1016/j.exger.2020.111138.

12. Jauch EC, Saver JL, Adams Jr HP, Bruno A, Connors JJ, Demaerschalk BM, et al. Guidelines for the early management depression of AIS patients still remain unclear. Further relevant study is needed.

In conclusion, common inflammatory cytokines including TNF- $\alpha$, IL-1 $1 \beta$, and IL-17 were related to cognition impairment, anxiety, and depression in AIS patients in this study. of patients with acute ischemic stroke: a guideline for healthcare professionals from the American Heart Association/American Stroke Association. Stroke 2013; 44: 870-947, doi: 10.1161/STR.0b013e318284056a.

13. Cumming TB, Churilov L, Linden T, Bernhardt J. Montreal cognitive assessment and mini-mental state examination are both valid cognitive tools in stroke. Acta Neurol Scand 2013; 128: 122-129, doi: 10.1111/ane.12084.

14. Zigmond AS, Snaith RP. The hospital anxiety and depression scale. Acta Psychiatr Scand 1983; 67: 361-370, doi: 10.1111/j.1600-0447.1983.tb09716.x.

15. Zhou L, Ma X, Wang W. Inflammation and coronary heart disease risk in patients with depression in China mainland: a cross-sectional study. Neuropsychiatr Dis Treat 2020; 16 : 81-86, doi: 10.2147/NDT.S216389.

16. Song L, Quan X, Su L, Wang K, Wang H, Wu L, et al. Inflammation and behavioral symptoms in preoperational glioma patients: is depression, anxiety, and cognitive impairment related to markers of systemic inflammation? Brain Behav 2020; 10: e01771, doi: 10.1002/brb3.1771.

17. Banks WA, Kastin AJ, Broadwell RD. Passage of cytokines across the blood-brain barrier. Neuroimmunomodulation 1995; 2: 241-248, doi: 10.1159/000097202.

18. Kohler $\mathrm{O}$, Krogh J, Mors $\mathrm{O}$, Benros ME. Inflammation in depression and the potential for anti-inflammatory treatment. Curr Neuropharmacol 2016; 14: 732-742, doi: 10.2174/ $1570159 \times 14666151208113700$.

19. Murakami Y, Saito K. Species and cell types difference in tryptophan metabolism. Int J Tryptophan Res 2013; 6: 4754, doi: 10.4137/IJTR.S11558.

20. Wang B, Lian YJ, Dong X, Peng W, Liu LL, Su WJ, et al. Glycyrrhizic acid ameliorates the kynurenine pathway in association with its antidepressant effect. Behav Brain Res 2018; 353: 250-257, doi: 10.1016/j.bbr.2018.01.024.

21. Cheng P, Zhang J, Wu Y, Liu W, Zhu J, Chen Z, et al. 5-HTTLPR polymorphism and depression risk in Parkinson's disease: an updated meta-analysis. Acta Neurol Belg 2020

22. Marcinkiewcz CA, Mazzone CM, D'Agostino G, Halladay LR, Hardaway JA, DiBerto JF, et al. Serotonin engages an anxiety and fear-promoting circuit in the extended amygdala. Nature 2016; 537: 97-101, doi: 10.1038/nature19318. 